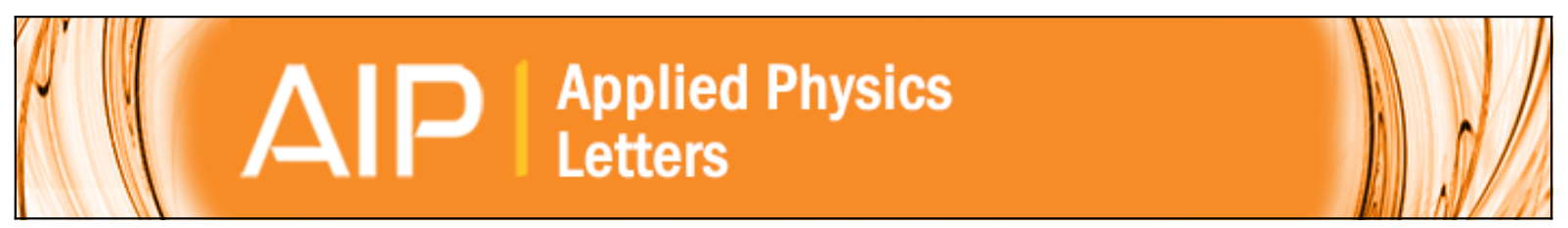

\title{
Microscopic scale investigation of piezoelectric properties of lead-free alkaline niobates
}

R. Machado, M. Sepliarsky, and M. G. Stachiotti

Citation: Applied Physics Letters 103, 242901 (2013); doi: 10.1063/1.4838115

View online: http://dx.doi.org/10.1063/1.4838115

View Table of Contents: http://scitation.aip.org/content/aip/journal/apl/103/24?ver=pdfcov

Published by the AIP Publishing

\section{Articles you may be interested in}

A monoclinic-tetragonal ferroelectric phase transition in lead-free $(\mathrm{KO} .5 \mathrm{Na} 0.5) \mathrm{NbO}-\mathrm{x} \% \mathrm{LiNbO} 3$ solid solution J. Appl. Phys. 111, 103503 (2012); 10.1063/1.4716027

High piezoelectric activity in ( $\mathrm{Na}, \mathrm{K}) \mathrm{NbO} 3$ based lead-free piezoelectric ceramics: Contribution of nanodomains Appl. Phys. Lett. 99, 062901 (2011); 10.1063/1.3624704

Piezoelectric properties of lithium modified silver niobate perovskite single crystals Appl. Phys. Lett. 92, 172905 (2008); 10.1063/1.2918837

Piezoelectric properties in perovskite 0.948 ( K $0.5 \mathrm{Na} 0.5$ ) Nb O $3-0.052$ Li Sb O 3 lead-free ceramics J. Appl. Phys. 100, 104108 (2006); 10.1063/1.2382348

Perovskite ( Na 0.5 K 0.5 ) $1-x$ ( Li Sb ) x Nb $1-x$ O 3 lead-free piezoceramics Appl. Phys. Lett. 88, 212908 (2006); 10.1063/1.2206554

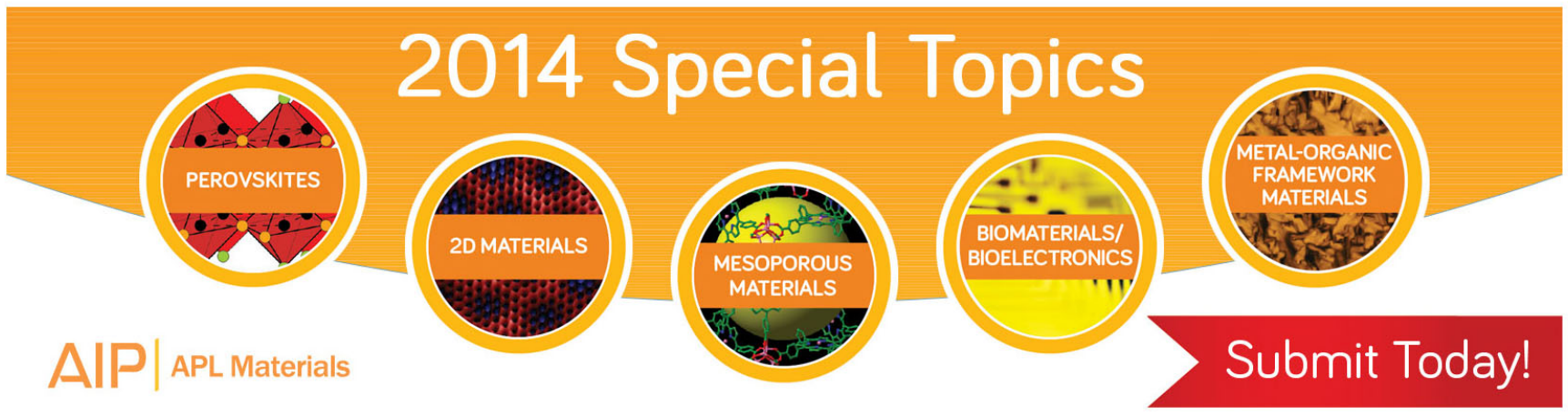




\title{
Microscopic scale investigation of piezoelectric properties of lead-free alkaline niobates
}

\author{
R. Machado, M. Sepliarsky, and M. G. Stachiotti \\ Instituto de Física Rosario, Universidad Nacional de Rosario, 27 de Febrero 210 Bis, 2000 Rosario, Argentina
}

(Received 1 October 2013; accepted 17 November 2013; published online 9 December 2013)

\begin{abstract}
A first-principles-based technique is used to investigate the electromechanical properties of lead-free alkaline niobates. In particular, we study the structural and piezoelectric properties of $\mathrm{Li}_{\mathrm{X}} \mathrm{K}_{1-\mathrm{x}} \mathrm{NbO}_{3}$ which is taken as a prototypical solid solution. We show that the system develops an orthorhombic to tetragonal polymorphic phase transition at room temperature for a $\mathrm{Li}$ concentration of $4.5 \%$. We showed that not only the phase coexistence region is relevant for the enhancement of the piezoelectric properties but also the off-center Li impurities play an important role in the improvement of the electromechanical response. (C) 2013 AIP Publishing LLC. [http://dx.doi.org/10.1063/1.4838115]
\end{abstract}

Piezoelectric properties play an important role for many electronic and functional devices. The most widely used piezoelectric materials are $\mathrm{PbZr}_{\mathrm{x}} \mathrm{Ti}_{1-\mathrm{x}} \mathrm{O}_{3} \quad$ (PZT)-based multi-component ceramics. However, the toxicity of lead oxide and its high vapor pressure during material processing may bring serious environmental problems. In recent years, especially after the publication of the grain-oriented $\mathrm{Na}_{0.5} \mathrm{~K}_{0.5} \mathrm{NbO}_{3}$-based ceramics, ${ }^{1}$ the researches on lead-free piezoelectrics have got much progress, ${ }^{2-6}$ and it is believed that the next generation of piezoceramics should be lead-free. ${ }^{7}$

The enhancement of the electromechanical response in lead-free materials is achieved by compositional engineering, where the composition of a solid-solution is optimized by bringing the material to the proximity of a structural instability such as a polymorphic phase transition or morphotropic phase boundary. ${ }^{6}$ Understanding the macroscopic properties of these materials from the atomic scale is then a very important challenge, not only because of the technological and social relevance of such materials but also because it would be of great help for the development of lead-free piezoceramics with superior properties. The main difficulty in simulating such materials using first-principles methods is their complexity since they exhibit chemical disorder on at least one, or in some cases two, of their cationic subnetworks, e.g., $\mathrm{Li}_{0.06}\left(\mathrm{Na}_{0.5} \mathrm{~K}_{0.5}\right)_{0.94} \mathrm{NbO}_{3}, \mathrm{Ba}_{0.06}\left(\mathrm{Na}_{0.5} \mathrm{Bi}_{0.5}\right)_{0.94}$ $\mathrm{TiO}_{3}, \mathrm{Ca}_{0.15} \mathrm{Ba}_{0.85} \mathrm{Zr}_{0.1} \mathrm{Ti}_{0.9} \mathrm{O}_{3}$, etc. For that reason, a multiscale approach based on the combination of ab-initio calculations with effective Hamiltonian ${ }^{8}$ or interatomic potential ${ }^{9}$ techniques is necessary.

Between the lead-free piezoelectrics, the alkaline niobates have been extensively investigated because of their good electromechanical properties and high Curie temperature. Piezoelectric properties comparable to the PZT system were announced by Saito et al. in $\mathrm{Na}_{0.5} \mathrm{~K}_{0.5} \mathrm{NbO}_{3}$ (NKN)based ceramics after appropriate chemical doping with $\mathrm{Li}$, Ta, and Sb. ${ }^{1}$ Further studies have shown that the only addition of an appropriate amount of Li ions into a ferroelectric NKN matrix is enough to produce a marked enhancement of the piezoelectric properties. ${ }^{10-12}$ The reinforcement of piezoelectric properties was attributed to the formation of a morphotropic phase boundary between orthorhombic and tetragonal phases in the $\mathrm{Li}$ composition range $0.05<x$ $<0.07$. $^{10,11}$ In this Letter, we report that ab-initio based simulations not only reproduce all the aforementioned features but also offer deep microscopic insights into the electromechanical properties of lead-free alkaline niobates. For that purpose, the $\mathrm{Li}_{\mathrm{x}} \mathrm{K}_{1-\mathrm{x}} \mathrm{NbO}_{3}$ solid solution is used as a prototypical material. We note that $\mathrm{KNbO}_{3}(\mathrm{KN})$ and $\mathrm{NKN}$ adopt the same series of crystal structures through all of their ferroelectric phases, ${ }^{13}$ and both are orthorhombic at room temperature. We find that the replacement of $\mathrm{K}$ by $\mathrm{Li}$ ions generates, at room temperature, a polymorphic phase transition at a Li concentration of $\approx 4.5 \%$. We show that the piezoelectric properties are strongly enhanced near the phase instability region, and that the off-center Li impurities play also an important role in the electromechanical response of the system.

We consider an interatomic potential approach based on a shell model. The details of the modeling procedure are presented in Ref. 14, where we showed that the combination of first-principles calculations with shell-model techniques is a suitable multiscale approach to investigate off-center $\mathrm{Li}$ impurities in $\mathrm{KNbO}_{3}$. In the present work, we use molecular-dynamics (MD) simulations to determine structural and piezoelectric properties of $\mathrm{Li}_{x} \mathrm{~K}_{1-x} \mathrm{NbO}_{3}(\mathrm{LKN})$ solid solutions. The simulations were done using the DL-POLY package. ${ }^{15}$ The runs were performed employing a Hoover constant- $(\sigma, \mathrm{T})$ algorithm with external stress set to zero; all cell lengths and cell angles were allowed to fluctuate. Periodic boundary conditions over 5000 atoms were considered. Piezoelectric coefficients $\left(\mathrm{d}_{\mathrm{ij}}\right)$ were determined by calculating the change in the elastic strain under applied electric fields. The coordinate system used for the orthorhombic phase was $[100]^{\mathrm{o}}=[100]^{\mathrm{c}}, \quad[010]^{\mathrm{o}}=[01-1]^{\mathrm{c}}$, $[001]^{\mathrm{O}}=[011]^{\mathrm{c}}$, that is means the polarization lies along the $[011]^{\mathrm{c}}$ direction. The tetragonal system is the same as the cubic one, and the polarization lies along the [001] direction.

The model for pure KN correctly reproduces the temperature-driven phase transition sequence experimentally observed; it adopts the series of phases cubic-tetragonalorthorhombic-rhombohedral (C-T-O-R) that is common to 


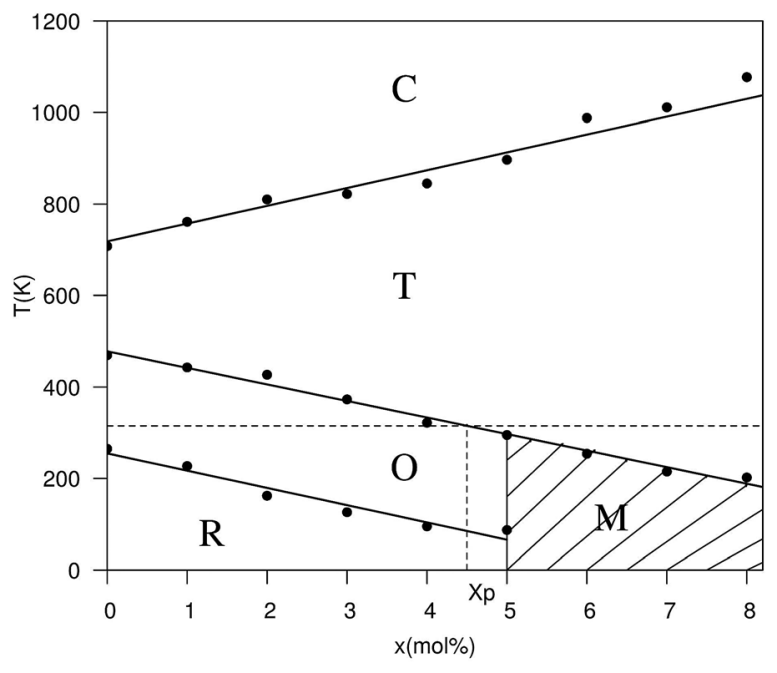

FIG. 1. Phase diagram of $\mathrm{Li}_{\mathrm{x}} \mathrm{K}_{1-\mathrm{x}} \mathrm{NbO}_{3}$ resulting from the MD simulations.

many perovskite ferroelectrics, including NKN. Details can be found in Ref. 14. Figure 1 shows the effect of the replacement of $\mathrm{K}$ by $\mathrm{Li}$ ions on the phase behavior of LKN solid solutions for $\mathrm{x}<8 \%$. This phase diagram clearly shows that $\mathrm{Li}$ impurities produce a decrease in the $\mathrm{R}-\mathrm{O}$ and $\mathrm{O}-\mathrm{T}$ transition temperatures, while the Curie temperature increases, producing an increase of the temperature range of stability of the tetragonal phase with increasing Li content. In the region of the phase diagram denoted by dashed lines ( $\mathrm{M}$ phase), the system does not polarize along any crystallographic direction; its polarization is oriented along arbitrary directions. It might be expected that at high Li concentrations, the Li displacements may show frustration of the $\mathrm{Li}$ in finding the optimal direction of off centering, which would be favorable for forming a relaxor ferroelectric. ${ }^{16}$ One important characteristic, regarding the phase diagram, is the existence of a structural instability at room temperature, due to the presence of an orthorhombic-to-tetragonal polymorphic phase transition at a Li concentration $\mathrm{x}_{\mathrm{p}}=4.5 \%$ (see the dotted lines in Figure 1). We observed, in our simulations, that near the polymorphic phase transition the system stabilizes either on orthorhombic, tetragonal, or monoclinic structures (i.e., with polarization along the (011) and (001) directions and in the (100) plane, respectively). It is believed that such kind of phase coexistence permits an almost continuous rotation of the polarization vector under an external electric field, producing an enhancement of the piezoelectric response. ${ }^{17}$ To provide microscopic insights about that point, we have determined the piezoelectric properties of the material for different $\mathrm{Li}$ contents.

One advantage of our approach is the possibility to calculate single crystal properties which are important to determine anisotropies. From symmetry considerations, there are five independent piezoelectric coefficients for the orthorhombic phase of LKN: the longitudinal $d_{33}$, the transverse $d_{31}$ and $\mathrm{d}_{32}$, and the shear components $\mathrm{d}_{15}$ and $\mathrm{d}_{24}$. In the tetragonal phase, $d_{31}=d_{32}$ and $d_{15}=d_{24}$. Figure 2 shows the $\mathrm{Li}$ concentration dependence of the piezoelectric coefficients at room temperature. The theoretical values for pure $\mathrm{KN}$ are $\mathrm{d}_{31}=-20, \mathrm{~d}_{32}=17, \mathrm{~d}_{33}=32, \mathrm{~d}_{15}=207, \quad$ and $\mathrm{d}_{24}=116$ $\mathrm{pC} / \mathrm{N}$, which are in reasonable good agreement with reported

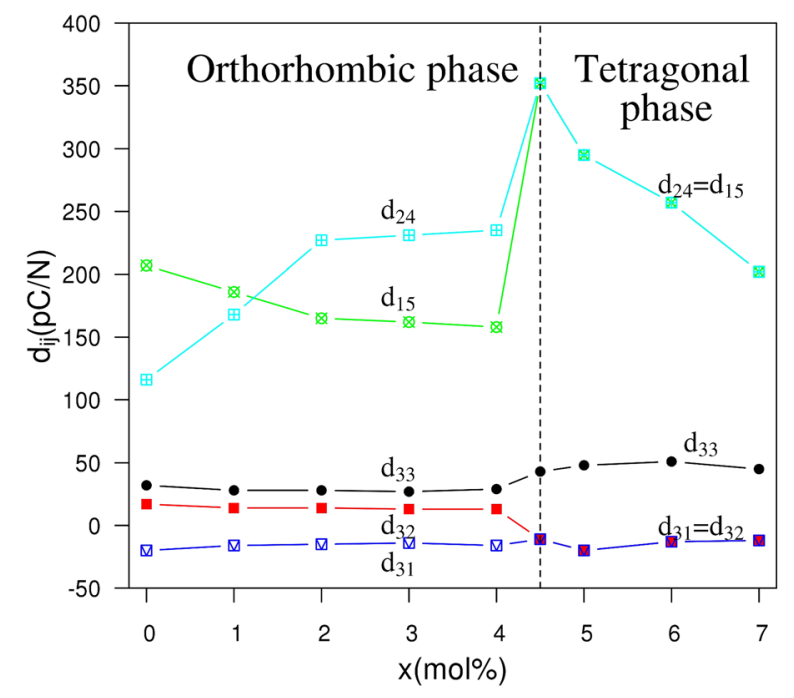

FIG. 2. Concentration dependence of piezoelectric coefficients $d_{i j}$ for $\mathrm{Li}_{\mathrm{x}} \mathrm{K}_{1-x} \mathrm{NbO}_{3}$. While the longitudinal and transverse coefficients practically do not depend on Li content, the shear components present quite strong concentration dependence.

experimental data. ${ }^{18-20}$ It is observed that the longitudinal and transverse coefficients do not depend on Li content. On the contrary, the shear components present quite strong concentration dependence. At low Li concentrations $(x<2 \%)$, the impurities strongly affect both shear components, $\mathrm{d}_{24}$ increases while $d_{15}$ decreases with $x$. This impurity effect seems to saturate and the shear components remain practically constant for $2 \%<\mathrm{x}<4 \%$. However, they strongly increase near the polymorphic phase transition. In the tetragonal phase, $d_{15}=d_{24}$ and they increase as the orthorhombic phase is approached, reaching a maximum value of $\approx 350$ $\mathrm{pC} / \mathrm{N}$ at the polymorphic phase transition. This behavior anticipates the incipient orthorhombic phase and reflects the softening of the lattice away from the polar [001] axis.

To determine the maximum deformation of the system along the direction of an external electric field, we calculated the longitudinal piezoelectric coefficient in an arbitrary direction, $\mathrm{d}_{33}^{*}(\varphi, \theta, \psi)$, where $\varphi, \theta$, are $\psi$ are the Euler angles. ${ }^{21,22}$ In the orthorhombic phase

$$
\begin{aligned}
d_{33}^{*}(\varphi, \theta)= & \cos (\theta)\left[\left(d_{15}+d_{31}\right) \sin ^{2}(\theta) \sin ^{2}(\varphi)\right. \\
& \left.+\left(d_{24}+d_{32}\right) \sin ^{2}(\theta) \cos ^{2}(\varphi)+d_{33} \cos ^{2}(\theta)\right],
\end{aligned}
$$

while in the tetragonal structure

$$
\mathrm{d}_{33}^{*}(\varphi, \theta)=\cos (\theta)\left[\left(\mathrm{d}_{15}+\mathrm{d}_{31}\right) \sin ^{2}(\theta)+\mathrm{d}_{33} \cos ^{2}(\theta)\right] .
$$

It is interesting to note that in the orthorhombic phase the maximum of $\mathrm{d}_{33}^{*}$ occurs either at $\varphi=0$ or at $\varphi=90$, depending on the relation between the first and second terms of Eq. (1). Figure 3(a) shows polar plots for the orientation dependence of piezoelectric coefficients $\mathrm{d}_{33}^{*}$ for pure KN (left) and LKN near the polymorphic phase transition (right). In the pure material, the maximum value of $\mathrm{d}_{33}^{*}$ is at $\varphi=90^{\circ}$ and $\theta=50.4^{\circ}$, while in the solid solution with $4 \% \mathrm{Li}$ the maximum $\mathrm{d}_{33}^{*}$ is at $\varphi=0^{\circ}$ and $\theta=52.2^{\circ}$, i.e., it is rotated $90^{\circ}$. For pure $\mathrm{KN}, \mathrm{d}_{15}$ is larger than $\mathrm{d}_{24}$, which makes the 


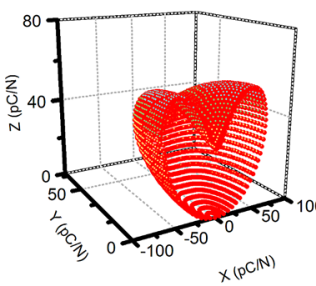

$x=0 \%$

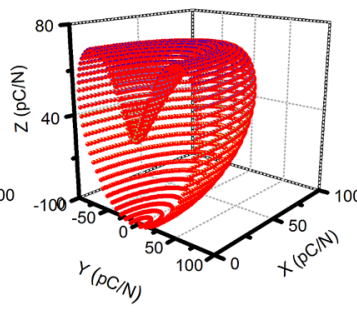

$x=4 \%$

(a)

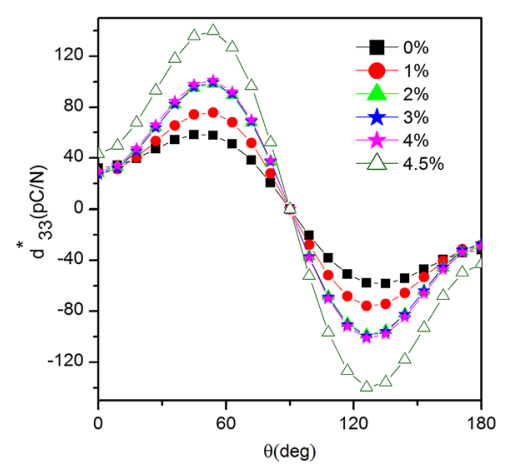

(c)

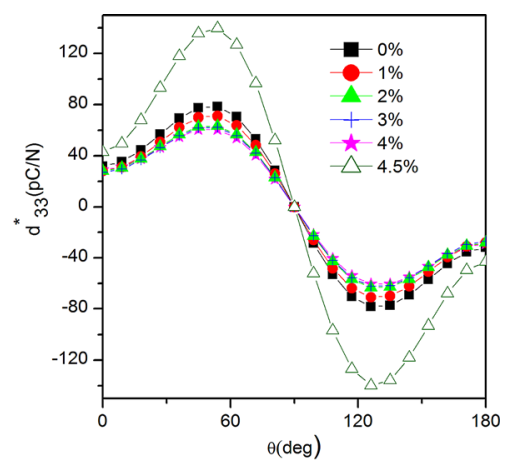

(b)

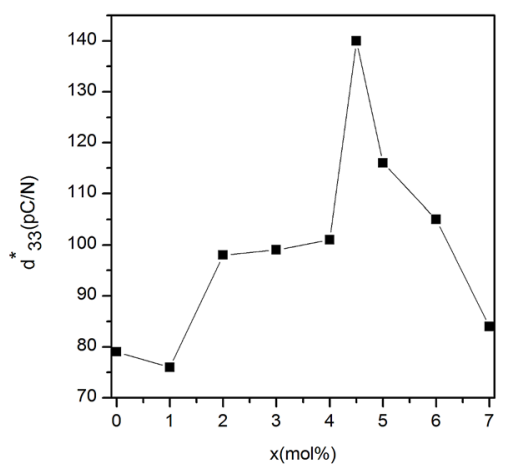

(d)
FIG. 3. (a) Orientation dependence of the piezoelectric coefficient $d_{33}^{*}$ in the orthorhombic phase of pure $\mathrm{KNbO}_{3}$ (left) and $\mathrm{Li}_{0.04} \mathrm{~K}_{0.96} \mathrm{NbO}_{3}$ near the polymorphic phase transition at room temperature (right). (b) and (c) The piezoelectric coefficient $\mathrm{d}_{33}^{*}$ in the orthorhombic phase as a function of angle $\theta$ at various Li contents: $\varphi=90^{\circ}$ (b) and $\varphi=0^{\circ}$ (c). (d) Maximum $\mathrm{d}_{33}^{*}$ as a function of $\mathrm{Li}$ content. maximum of $\mathrm{d}_{33}^{*}$ lie in the orthorhombic XZ plane, which contains the $[111]^{\mathrm{c}}$ and $[100]^{\mathrm{c}}$ cubic directions. This is because the room temperature state of the pure material is near the R-O phase transition (see Figure 1), so it is easier to produce a rhombohedral deformation with an electric field along the $[100]^{\circ}$ direction than a tetragonal deformation with an electric field in the $[010]^{\circ}$ direction. The addition of $\mathrm{Li}$ brings the system close to the orthorhombic-tetragonal polymorphic phase transition, that is $d_{24}$ increases while $d_{15}$ decreases, changing the maximum of $\mathrm{d}_{33}^{*}$ to the $\mathrm{YZ}$ cubic plane. To better understand that behavior, we show in Figure 3 the values of $\mathrm{d}_{33}^{*}$ as a function of $\theta$ for $\varphi=90^{\circ}$ (Figure 3(b)) and $\varphi=0^{\circ}$ (Figure 3(c)). It is clear that the addition of $\mathrm{Li}$ produces the decrease (increase) of $\mathrm{d}_{33}^{*}$ for $\varphi=90^{\circ}\left(\varphi=0^{\circ}\right)$. With the constrain of $\varphi=90^{\circ}$, the maximum value of $\mathrm{d}_{33}^{*}$ is always at $\theta=50.4^{\circ}$, so it deviates from the $[111]^{\mathrm{c}}$ direction by almost $15^{\circ}$ (the $[111]^{\mathrm{c}}$ corresponds to $\varphi=90^{\circ}$ and $\left.\theta=\pi / 2-\operatorname{atan}\left(2^{0.5}\right) \approx 35.26^{\circ}\right)$. For $\varphi=0^{\circ}$, the maximum is at $\theta=50.4^{\circ}$ for $\mathrm{x}=0$ and $\mathrm{x}=0.01$, and $\theta=52.2^{\circ}$ for $2 \% \leq \mathrm{x} \leq 4.5 \%$, which are close to the $[001]^{\mathrm{c}}$ direction $\left(\varphi=0^{\circ}\right.$ and $\left.\theta=45^{\circ}\right)$. In both cases, $\mathrm{d}_{33}^{*}$ shows a significant increment at the polymorphic phase transition $(x=4.5 \%)$. We plot in Figure 3(d) the behavior of the maximum value adopted by $\mathrm{d}_{33}^{*}\left(\mathrm{~d}_{33 \text { max }}^{*}\right)$ as a function of Li concentration. There is an increment of about $25 \%$ between pure $\mathrm{KN}$ and the material doped with $2 \%$ of $\mathrm{Li}$, while $\mathrm{d}_{33 \text { max }}^{*}$ practically does not change for $2 \%<\mathrm{x}<4 \%$. The effective piezoelectric constant $\mathrm{d}_{33 \max }^{*}$ shows an evident peak $(75 \%$ of increment) at the polymorphic phase transition, in qualitative agreement with experimental data of $\mathrm{Li}$ doped NKN ceramics. $^{10,11}$

To investigate the polarization rotation mechanism at stronger fields, we calculated the longitudinal strains as a function of the field intensity for electric fields applied along the $[001]^{\mathrm{c}}$ direction. The results for different Li contents are shown in Figure 4(a). The systems display the characteristic linear response behavior in the low field regime, where the slopes of the curves increase with the Li content. If the electric field is further increased, the strain response displays a strong jump, which is a signature of a field induced (orthorhombic to tetragonal) phase transition. To better appreciate the transitions, we plot in Figure 4(b) the angle between the polarization vector and the applied electric field as a function of field intensity. That figure shows that the polarization vector rotates completely from the $[011]^{\mathrm{c}}$ to the $[001]^{\mathrm{c}}$ direction when the applied electric field exceeds a certain critical value $E_{R}$ (this field coincides with the field required to produce the jump in the strain response). It is clear that the critical field $\mathrm{E}_{\mathrm{R}}$ depends on $\mathrm{Li}$ concentration. The inset of Figure 4(b) shows that $E_{R}$ decreases monotonously as $\mathrm{x}$ increases, which indicates that the off-center $\mathrm{Li}$ impurities favor the rotation of the polarization vector under the applied electric field.

To better understand the strain response at a microscopic level, we have performed a more detailed characterization of the polarization rotation process in terms of local polarizations, distinguishing unit cells centered on $\mathrm{Li}$ and $\mathrm{K}$ ions. Figure 4(c) shows the angles between the applied electric field and the polarization vectors of the $\mathrm{Li}$ and $\mathrm{K}$ sublattices for the case $x=2 \%$. It is clear that the $\mathrm{Li}$ sublattice is advanced with respect to the $\mathrm{K}$ cells at most of the rotation process. For example, for an electric filed $E=E_{R} / 2$, the rotation angle of the $\mathrm{Li}$ sublattice is ahead by $\delta \theta \approx 10^{\circ}$, that is $30 \%$. The value of $\delta \theta$ practically does not depend on Li concentration. When the applied electric field reaches the critical value $E_{R}$, the induced phase transition is produced suddenly by the abrupt and simultaneous rotation of the two sublattices. The scenario described above indicates that the 


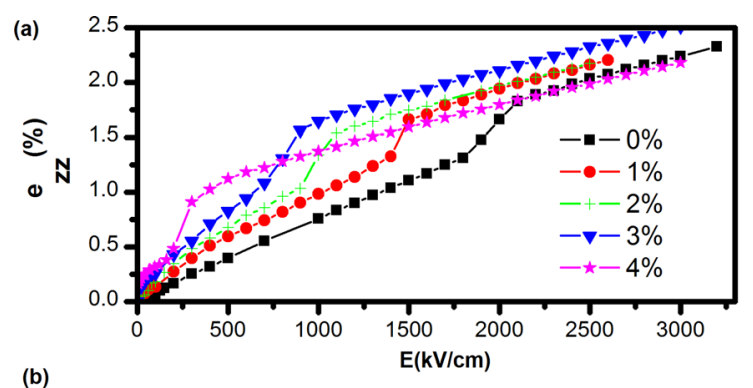

(b)

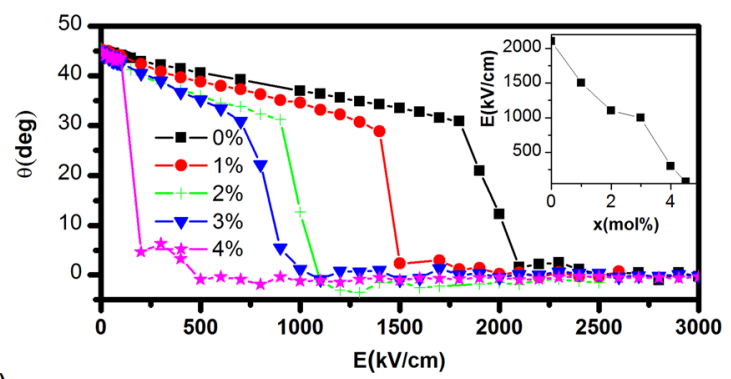

(c)

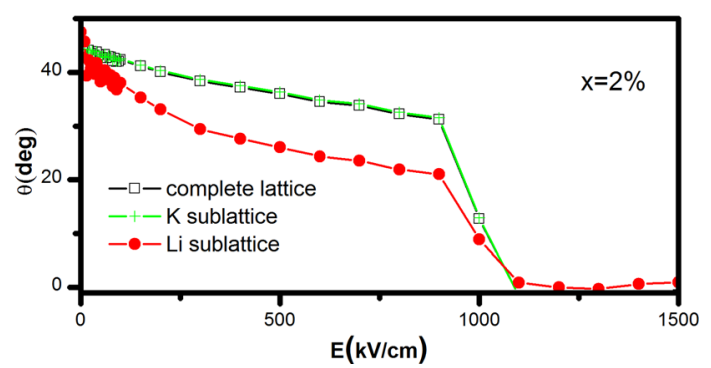

FIG. 4. (a) Strain response as a function of field intensity for various Li contents at room temperature. The electric field was applied along the [001] $]^{\mathrm{c}}$ direction. (b) Angle between the polarization vector and the applied electric field along the $[001]^{\mathrm{c}}$ direction as a function of the field intensity for various $\mathrm{Li}$ contents. (c) Angle between the applied electric field and the polarization vectors of $\mathrm{Li}$ and $\mathrm{K}$ sublattices as a function of the field intensity for the case $\mathrm{x}=2 \%$.

off-center Li impurities have the property to "drag" the $\mathrm{K}$ sublattice in the rotation process, improving the electromechanical response of the system. The quite different response of the two sublattices to an external electric field might be related to their dynamical behavior which is mainly relaxational for the off-center Li ions. ${ }^{14}$ Further studies will be necessary, however, to better clarify that point and also to understand the role of Li-Li correlations in the piezoelectric response of the system.

In summary, we showed that the combination of firstprinciples calculations with shell-model techniques is a suitable multiscale approach to investigate the piezoelectric properties of $\mathrm{Pb}$-free solid solutions. In particular, we found that the replacement of $\mathrm{K}$ by $\mathrm{Li}$ ions in $\mathrm{KNbO}_{3}$ generates a polymorphic phase transition at room temperature for a $\mathrm{Li}$ concentration of $4.5 \%$. We showed that not only the phase coexistence region is relevant to produce the enhancement of the piezoelectric properties but also the off-center Li impurities play an important role in the electromechanical response of the system.

We acknowledge computing time at the CCT-Rosario Computational Center. This work was sponsored by Consejo Nacional de Investigaciones Científicas y Tecnológicas (CONICET) and Agencia Nacional de Promoción Científica y Tecnológica (ANPCyT) de la República Argentina. M.G.S. thanks for the support from Consejo de Investigaciones de la Universidad Nacional de Rosario (CIUNR).

${ }^{1}$ Y. Saito, H. Takao, T. Tani, T. Nonoyama, K. Takatori, T. Homma, T. Nagaya, and M. Nakamura, Nature 432, 84 (2004).

${ }^{2}$ M. Maerder, D. Damjanovic, and N. Setter, J. Electroceram. 13, 385 (2004).

${ }^{3}$ T. Takenaka and H. Nagata, J. Eur. Ceram. Soc. 25, 2693 (2005).

${ }^{4}$ P. K. Panda, J. Mater. Sci. 44, 5049 (2009).

${ }^{5}$ Y. Lu and Y. Li, J. Adv. Dielectr. 1, 269 (2011).

${ }^{6}$ S. O. Leontsev and R. E. Eitel, Sci. Technol. Adv. Mater. 11, 044302 (2010).

${ }^{7}$ E. Cross, Nature 432, 24 (2004).

${ }^{8}$ D. Vanderbilt, Curr. Opin. Solid State Mater. Sci. 2, 701 (1997).

${ }^{9}$ M. Sepliarsky, A. Asthagiri, S. R. Phillpot, M. G. Stachiotti, and R. L. Migoni, Curr. Opin. Solid State Mater. Sci. 9, 107 (2005).

${ }^{10}$ Y. Guo, K. Kakimoto, and H. Ohsato, Appl. Phys. Lett. 85, 4121 (2004).

${ }^{11}$ E. Hollenstein, M. Davis, D. Damjanovic, and N. Setter, Appl. Phys. Lett. 87, 182905 (2005).

${ }^{12}$ P. Zhao, B.-P. Zhang, and J.-F. Li, Appl. Phys. Lett. 90, 242909 (2007).

${ }^{13}$ H. J. Trodahl, N. Klein, D. Damjanovic, N. Setter, B. Ludbrook, D. Rytz, and M. Kuball, Appl. Phys. Lett. 93, 262901 (2008).

${ }^{14}$ R. Machado, M. Sepliarsky, and M. G. Stachiotti, Phys. Rev. B 86, 094118 (2012).

${ }^{15} \mathrm{~W}$. Smith and T. R. Forester, DL-POLY is a Package of Molecular Simulation Routines (Daresbury and Rutherford Appleton Laboratory, Daresbury, UK, 1996).

${ }^{16}$ D. I. Bilc and D. J. Singh, Phys. Rev. Lett. 96, 147602 (2006).

${ }^{17}$ H. X. Fu and R. E. Cohen, Nature 403, 281 (2000).

${ }^{18}$ E. Wiesendanger, Ferroelectrics 6, 263 (1973).

${ }^{19}$ P. Gunter, Jpn. J. Appl. Phys., Part 1 16, 1727 (1977).

${ }^{20}$ S. Wada, K. Muraoka, H. Kakemoto, T. Tsurumi, and H. Kumagai, Jpn. J. Appl. Phys., Part 1 43, 6692 (2004).

${ }^{21}$ F. J. Nye, Physical Properties of Crystals (Oxford Science Publications, New York, 1985).

${ }^{22}$ L. Liang, Y. L. Li, S. Y. Hu, L.-Q. Chen, and G.-H. Lu, J. Appl. Phys. 108, 094111 (2010). 